\title{
Coordinated lonospheric Reconstruction CubeSat Experiment (CIRCE), In situ and Remote lonospheric Sensing (IRIS) suite
}

\author{
Gemma D. R. Attrill ${ }^{1, *}$, Andrew C. Nicholas ${ }^{2}$, Graham Routledge ${ }^{3}$, Junayd A. Miah ${ }^{1}$,
} Dhiren O. Kataria ${ }^{4}$, Cathryn N. Mitchell ${ }^{5}$, Robert J. Watson ${ }^{5}$, James Williams ${ }^{6}$, Alex Agathanggelou ${ }^{3}$, Charles M. Brown ${ }^{2}$, Scott A. Budzien ${ }^{2}$, Tobias Carman ${ }^{3}$, Rahil Chaudery ${ }^{4}$, Kenneth F. Dymond ${ }^{2}$, Ted T. Finne ${ }^{2}$, Alex Fortnam ${ }^{3}$, Bruce Fritz ${ }^{2}$, Alex Hands ${ }^{7}$, Peter J. Marquis ${ }^{2,8}$, Sean Murphy ${ }^{1}$, Talini Pinto-Jayawardena ${ }^{5,9}$, Duncan Rust ${ }^{4}$, Keith A. Ryden ${ }^{7}$, Dave Schofield ${ }^{6}$, Andrew W. Stephan ${ }^{2}$, Kevin Wiggins ${ }^{6}$, and Craig Underwood ${ }^{7}$

${ }^{1}$ Defence Science and Technology Laboratory, Porton Down, Salisbury, Wiltshire SP4 0JQ, UK

${ }^{2}$ U.S. Naval Research Laboratory, 4555 Overlook Ave, Washington, DC 20375, USA

${ }^{3}$ Defence Science and Technology Laboratory, Portsdown West, Portsdown Hill Road, Fareham PO17 6AD, UK

${ }^{4}$ Mullard Space Science Laboratory, University College London, Surrey RH5 6NT, UK

${ }^{5}$ Department of Electronic and Electrical Engineering, University of Bath, Bath BA2 7AY, UK

${ }^{6}$ Surrey Satellite Technologies Limited, Tycho House, 20 Stephenson Rd, Guildford GU2 7YE, UK

${ }^{7}$ University of Surrey, Stag Hill, University Campus, Guildford GU2 7XH, UK

${ }^{8}$ Now at NASA Wallops Space Flight Center, Wallops Island, VA 23337, USA

9 Athena Space Ltd, Torquay, Devon TQ2 7TD, UK

Received 30 June 2020 / Accepted 10 November 2020

\begin{abstract}
The UK's Defence Science and Technology Laboratory (Dstl) is partnering with the US Naval Research Laboratory (NRL) on a joint mission to launch miniature sensors that will advance space weather measurement and modelling capabilities. The Coordinated Ionospheric Reconstruction Cubesat Experiment (CIRCE) comprises two 6U cube-satellites that will be launched into a near-polar low earth orbit (LEO), targeting $500 \mathrm{~km}$ altitude, in 2021. The UK contribution to CIRCE is the In situ and Remote Ionospheric Sensing (IRIS) suite, complementary to NRL sensors, and comprising three highly miniaturised payloads provided to Dstl by University College London (UCL), University of Bath, and University of Surrey/ Surrey Satellite Technology Ltd (SSTL). One IRIS suite will be flown on each satellite, and incorporates an ion/neutral mass spectrometer, a tri-band global positioning system (GPS) receiver for ionospheric remote sensing, and a radiation environment monitor. From the US, NRL have provided two 1U Triple Tiny Ionospheric Photometers (Tri-TIPs) on each satellite (Nicholas et al., 2019), observing the ultraviolet $135.6 \mathrm{~nm}$ emission of atomic oxygen at night-time to characterize the two-dimensional distribution of electrons.
\end{abstract}

Keywords: ionosphere / space weather / cube-satellite

\section{Introduction}

The Coordinated Ionospheric Reconstruction Cubesat Experiment (CIRCE) will characterise a region of the space environment, the ionosphere, which is important for a range of defence and civil applications and can impact global navigation satellite system (GNSS), communications and sensing technology. Nicholas et al. (2019) provides an overview of the CIRCE mission.

\footnotetext{
*Corresponding author: gdrattrill@dstl.gov.uk
}

The thermosphere is a region of the neutral atmosphere, from $\sim 85 \mathrm{~km}$ to $\sim 500 \mathrm{~km}$ altitude, heated by solar ultraviolet (UV) radiation, where the temperature gradient remains positive. It is characterized by high temperature and large variability, in response to changes in solar UV radiation and solar-driven geomagnetic activity. The thermospheric temperature eventually becomes almost constant at a value that varies with time, but is generally over $1000 \mathrm{~K}$. This is the hottest part of the atmosphere. Here, mixing is inhibited by the positive temperature gradient, and the various components separate under gravity so that the composition varies with altitude (Hargreaves, 1992). The composition of the lower thermosphere is primarily 
$\mathrm{N}_{2}$ and $\mathrm{O}_{2}$, but in the upper thermosphere atomic oxygen $(\mathrm{O})$ becomes the dominant gas, due to the importance of photodissociation (a chemical reaction that degrades compounds by bombarding the molecule with photons) and molecular diffusion at high altitude (Solomon \& Roble, 2015).

The ionosphere is part of the upper atmosphere formed by the ionisation of gases including $\mathrm{N}_{2}, \mathrm{O}_{2}$, and $\mathrm{O}$. At middle and low latitudes, the primary source of energy comes from solar radiation at extreme ultra-violet (EUV) and X-ray wavelengths. At high latitudes, a significant source is energetic particles, in the form of electrons associated with the aurora, and primarily protons emitted from the sun during some solar flares. Once ionised, the ions and electrons recombine and react with other gaseous species to produce other ions. The net concentration of free electrons (referred to as the electron density) therefore depends on the relative speed of these production and loss processes, which also includes the mean drift velocity of the electrons (see e.g. Schunk \& Nagy, 2009 for further details).

The ionosphere contains significant numbers of free electrons and positive ions, though the medium as a whole is electrically neutral. Hargreaves (1992) describes the different ionospheric regions with the following daytime electron densities:

- D region, 60-90 km: $10^{8}-10^{10} \mathrm{~m}^{-3}$;

- E region, 105-160 km: several $10^{11} \mathrm{~m}^{-3}$;

- F1 region, 160-180 km: several $10^{11}-10^{12} \mathrm{~m}^{-3}$;

- F2 region, maximum variable around $300 \mathrm{~km}$ : up to several $10^{12} \mathrm{~m}^{-3}$

During the day, the intensity of ionising radiation varies with the elevation of the Sun, and the electron density responds accordingly. With the removal of the solar source of radiation, the $\mathrm{D}$ and $\mathrm{F} 1$ regions vanish at night, and the $\mathrm{E}$ region becomes much weaker $\left(\sim 5 \times 10^{9} \mathrm{~m}^{-3}\right)$ as the electron density decays. The F2 region persists though at reduced intensity. CIRCE will fly at an altitude of $500 \mathrm{~km}$, and will focus on characterising the upper part of the $\mathrm{F}$ region.

As discussed above, the level of solar activity, time of day, and also seasonal variations have a marked impact on typical electron density profiles. The interested reader is referred to Figure 7.15 in Hargreaves (1992), which shows data from Millstone Hill in the USA under a variety of conditions.

The total electron content (TEC) is defined as the integrated value of electron concentration along the line of sight from a transmitter to a receiver. The TEC in the ionosphere is modified by multiple factors, including the changing solar EUV radiation, geomagnetic storms, and the atmospheric waves that propagate up from the lower atmosphere. TEC is measured in electrons per square meter. By convention, 1 TEC Unit $(\mathrm{TECU})=10^{16}$ electrons $\mathrm{m}^{-2}$. Vertical TEC values in Earth's ionosphere typically range from a few to several hundred TECU. Though, for an extreme, Carrington-level space weather event, Hapgood et al. (2018) estimate that TEC at middle latitudes could exceed 1000 TEC units.

The propagation of radio waves is affected by the physical characteristics of the medium through which they propagate. To a first approximation, the electron density, $\mathrm{n}_{\mathrm{e}}$, of the ionospheric plasma is related to the plasma frequency, $f$, by $f=9 \sqrt{ } n_{e}$. In the lower ionosphere (D region), where the electron density, and electron-neutral collision frequency are relatively large, radio absorption prevails. Fadeouts in high-frequency (HF; 3-30 $\mathrm{MHz}$ ) radio propagation result from abnormally strong absorption in the ionosphere occurring due to enhanced electron density in the D region. The phase and group delays, reflection, and refraction that the entire ionosphere produces (especially near the maximum ionisation region, within the F2 layer) can affect the propagation of radio signals across a significant proportion of the electromagnetic spectrum, including into the microwave region.

In addition to this background variability, space weather effects such as solar flares and coronal mass ejections (CMEs, e.g. Phillips, 1992), drive more dynamic and significant changes to the behaviours of our near-Earth space environment. For example, the high frequency fadeouts discussed above, due to enhanced electron density in the D region, were found to occur in association with solar flares (Dellinger, 1937). These were later attributed primarily to X-ray emission from the flare, and are part of the category of sudden ionospheric disturbances. Changes in the Earth's geomagnetic field as a result of CMEs interacting with the magnetosphere are reflected in disturbed ionospheric behaviour. The work of Dungey (1961) showed how, in the case of the magnetosphere, reconnected ("open") magnetic field lines thread its outer boundary (the magnetopause) and so connect the geomagnetic field to the interplanetary magnetic field, allowing under the correct conditions, energy, mass, and momentum to be transferred from the solar wind and appropriately-oriented CMEs, into the magnetosphere and down into the ionosphere and thermosphere (Lockwood, 2016). It is worth highlighting that whilst some aspects of space weather, for example sudden ionospheric disturbances and solar radio bursts, act on a global scale and can impact the whole of the Earth's sunlit hemisphere at once (Nita et al., 2002; Pick, 2006), other ionospheric disturbances (discussed below) do not impact the entire globe in a uniform manner (e.g. Basu \& Groves, 2001).

Radio signal delays (corresponding to ranging errors) can be caused by changes in the ionospheric electron density, or gradients in the bulk ionosphere total electron content (TEC). Such gradients include travelling ionospheric disturbances (TIDs, e.g. Altadill et al., 2020). Small-scale irregularities in the electron density cause Fresnel scattering of radio signals, and fieldaligned structures such as auroral arcs and equatorial plumes cause diffraction effects known as scintillation (Basu et al., 1988). Scintillation can lead to signal fade, disruption and distortion of electromagnetic waves affecting a wide range of systems. Understanding the impact of a given aspect of space weather requires an understanding of the system in question, which is where the field of space weather science must work in partnership with the realm of engineering (Cannon et al., 2013).

There is growing demand across many sectors of the UK for access to an improved level of space awareness. This is necessary both on the ground, for example to predict geomagnetically induced currents in the national grid (Beggan et al., 2013), as well as in space. The latter includes information to prevent collisions in space, mitigate space weather impacts and assist in anomaly resolution, including that of radio frequency interference. 
The objectives of the CIRCE mission are to accurately characterize the dynamic ionosphere by:

- making in situ ionospheric particle and radiation measurements combined with remote-sensing of GPS signals to map the ionosphere, and;

- providing tomographic specification of electron density versus altitude derived from ultraviolet (UV) observations of the ionosphere from multiple cubesats and different view angles.

A unique aspect of the CIRCE mission is the ability to characterise the same volume of space within a very short return period, enabled by the constellation of two near-identical spacecraft actively maintaining a lead-follow configuration in the same low Earth orbit, separated by $250-500 \mathrm{~km}$. A single satellite in LEO has a $\sim 90$ min revisit period, a multi-satellite constellation would improve on this, but it is the dual-nature of the two relatively closely-spaced CIRCE satellites that is anticipated to provide unique insights into the temporal dynamics associated with specific ionospheric structures.

\section{CIRCE overview}

\subsection{The CIRCE satellite buses}

CIRCE comprises two $6 \mathrm{U}^{1}(2 \mathrm{U} \times 3 \mathrm{U})$ CubeSat buses provided by Blue Canyon Technologies (see Figs. 1 and 2), to fly a total of 10 payloads. The two satellites will fly lead/trail in tandem, in coplanar orbit to collect ionospheric measurements optimized for tomographic reconstruction. Launch will be facilitated by the US Department of Defense (DoD) Space Test Program, the objective is a near-polar orbit $500 \mathrm{~km}$ $( \pm 10 \mathrm{~km})$, with an inclination of $90^{\circ}\left( \pm 5^{\circ}\right)$.

\subsection{Tri-TIP - overview}

The US NRL provides two Triple Tiny Ionospheric Photometers (Tri-TIPs) for each satellite, observing the ultraviolet $135.6 \mathrm{~nm}$ emission of atomic oxygen at night-time (see Fig. 3). The primary objective is to characterize the twodimensional distribution of electrons.

The methodology used to reconstruct the night-time ionosphere employs continuous UV photometry from four distinct viewing angles in combination with an additional data source, such as in situ plasma density measurements, with advanced image space reconstruction algorithm tomography techniques.

Only a brief overview of the US Tri-TIP payloads is provided here. The Tri-Tip sensor is so named because it uses three photomultiplier tubes (PMTs), one for the UV signal and two to assess background signals. The reader is referred to Nicholas et al. (2019) for further details on Tri-TIP, including calibration information.

\subsection{IRIS payloads - overview}

The UK contribution to CIRCE is the In situ and Remote Ionospheric Sensing (IRIS) suite. IRIS provides in situ

\footnotetext{
${ }^{1} 1$ Unit $(1 \mathrm{U})$ is $10 \mathrm{~cm} \times 10 \mathrm{~cm} \times 10 \mathrm{~cm}$.
}

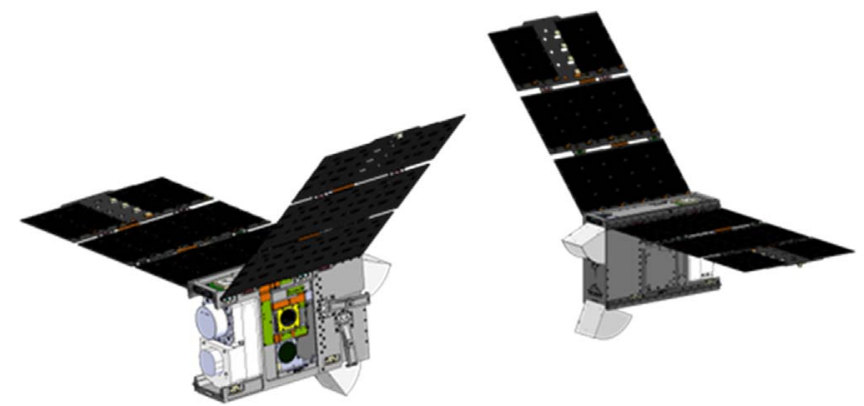

Fig. 1. Computer aided design (CAD) models illustrating the CIRCE concept (courtesy Blue Canyon Technologies).

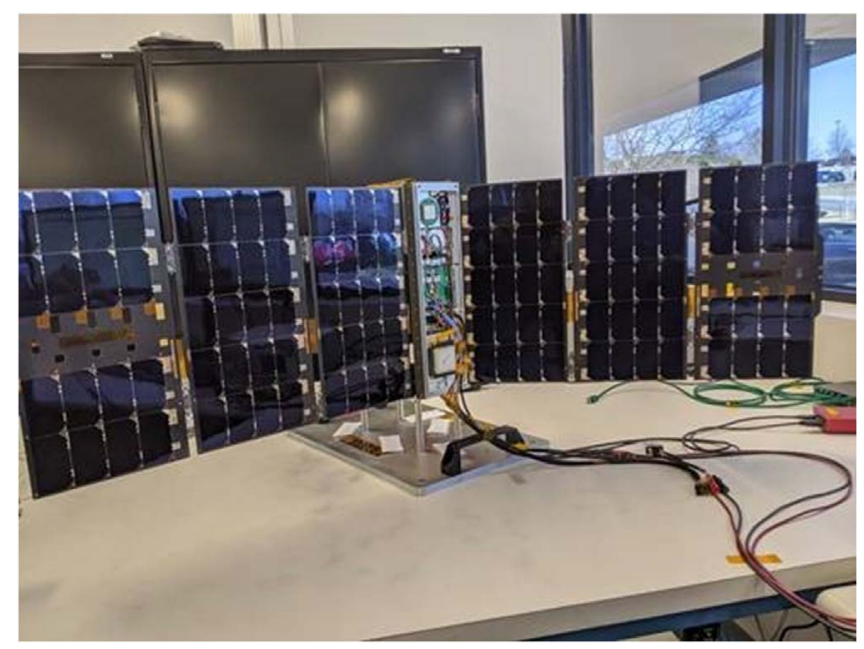

Fig. 2. CIRCE lead satellite bus, complete with solar arrays (courtesy Blue Canyon Technologies).

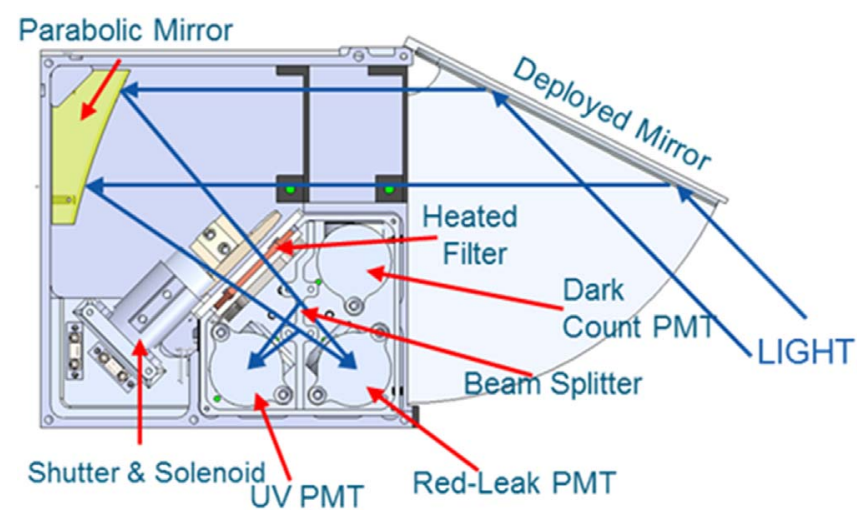

Fig. 3. Top-down view of the optical layout of the Tri-TIP sensor. (From Nicholas et al., 2019, their Fig. 2).

ionospheric particle and radiation measurements combined with remote-sensing of GPS signals to map the ionosphere.

The three IRIS payloads have been carefully selected to complement the US NRL's Tri-TIPs, and to showcase the capabilities of UK academia: University College London/Mullard Space Science Laboratory (provided the Ion and Neutral Mass Spectrometer; INMS), University of Bath (provided the 
Table 1. Key attributes of the IRIS payloads.

\begin{tabular}{lccc}
\hline & INMS & TOPCAT II & RadMon 3.0 \\
\hline Mass & $220 \mathrm{~g}$ & $145 \mathrm{~g}$ & $200 \mathrm{~g}$ \\
Power & $0.9 \mathrm{~W}$ & $120 \mathrm{~g} \mathrm{(antenna)}$ & $1.8 \mathrm{~W}$ \\
Data interface & RS232 & $2.3 \mathrm{~W}$ & RS422 \\
Accommodation & Ram direction & Based on CCSDS & Antenna externally mounted
\end{tabular}
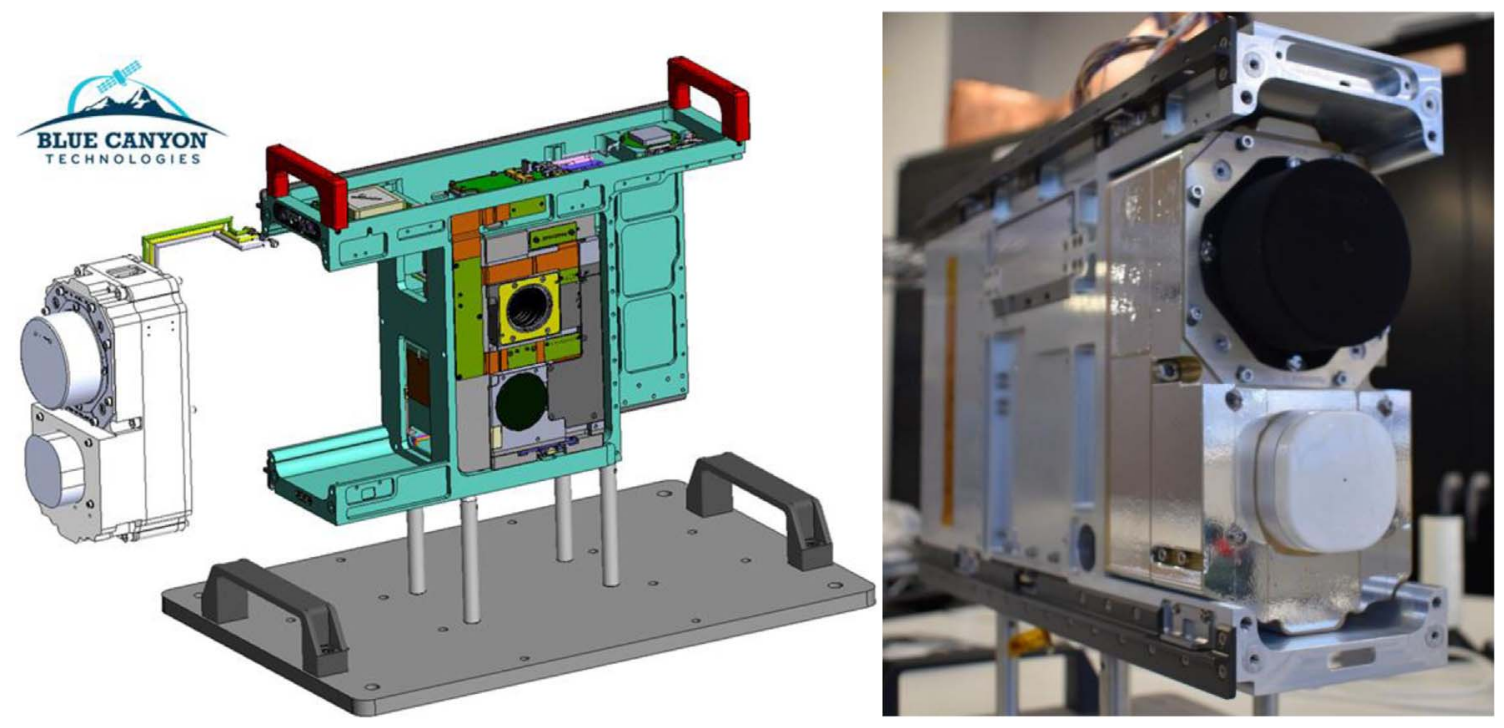

Fig. 4. CAD model (left panel), and photograph (right) showing how IRIS is integrated into the CIRCE bus chassis (courtesy Blue Canyon Technologies).

TOPside ionosphere and plasmasphere Computer Assisted Tomography tri-band GPS receiver; TOPCAT II), and Surrey Satellite Technology Ltd/University of Surrey (provided a compact radiation monitor; RadMon 3.0). The individual IRIS payloads will provide contextual environmental information to CIRCE, and enrich the data that can be derived from the US payloads. Table 1 details some key attributes of the IRIS payloads.

A key achievement of the UK contribution has been the rapid development timescale of IRIS, from concept through to flight model payload delivery of two complete suites, which was completed in just 1 year.

\section{CIRCE payload laydown and manoeuvers}

Figure 4 shows how the UK payloads have been integrated to form IRIS, and where they are located within the satellite bus.

The CIRCE buses will conduct manoeuvres without propulsion, using differential drag to achieve and maintain a baseline in-track separation $(250-500 \mathrm{~km})$.

Figure 5 depicts the lead and trail spacecraft configuration that will provide the Tri-TIP payloads with the best geometry for the night side observations of the ionosphere that will support tomographic reconstruction. Tri-TIP is configured for four look directions:

- Lead spacecraft Tri-TIP 1 door/mirror deploys to provide a look direction that is $17^{\circ}$ down from the horizon and away from the direction of spacecraft motion.

- Lead spacecraft Tri-TIP 2 door/mirror deploys to provide a look direction that is $45^{\circ}$ down from the horizon away from the direction of spacecraft motion.

- Trail spacecraft Tri-TIP 1 door mirror deploys to provide a view straight down (nadir), light does not reflect off the deployed mirror prior to entering instrument.

- Trail spacecraft Tri-TIP 2 door/mirror deploys to provide a look direction that is $45^{\circ}$ down from the horizon along the direction of spacecraft motion.

The Tri-TIP design uses replaceable door stops to control the angle of door/mirror deployment. The nadir viewing instrument deploys beyond $100^{\circ}$, but when stowed provides shielding to the instrument aperture from dust and contamination.

The UK INMS payloads are optimised for measurements in the ram direction. In order to maximise INMS instrument data collection, at the beginning of the day side portion of an orbit, the trail satellite will perform a rotation of $180^{\circ}$, allowing the IRIS instruments (on both satellites) to all face in the ram 


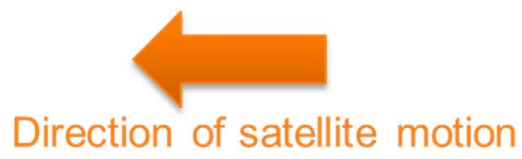

\section{Leading Satellite}

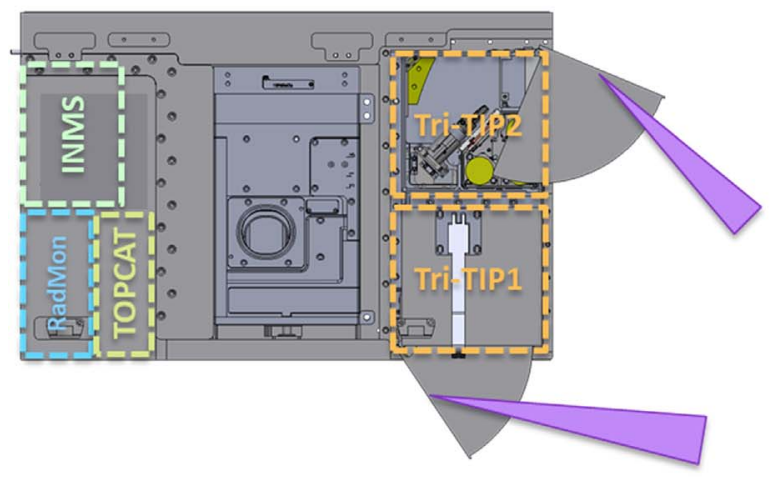

Trailing Satellite

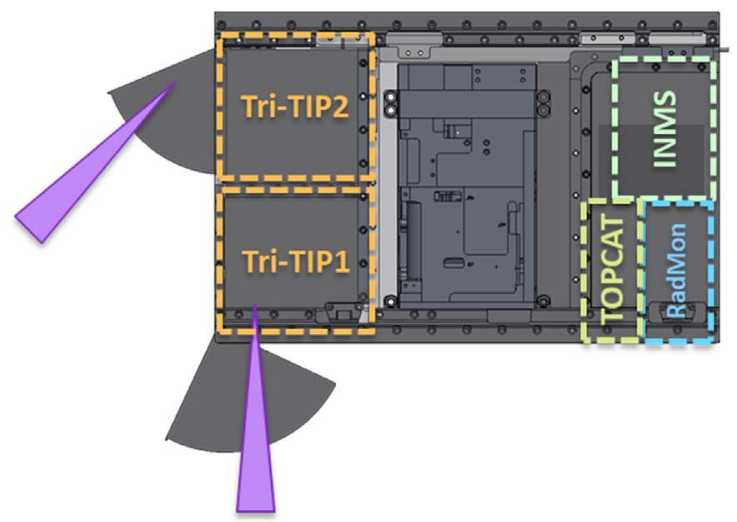

Fig. 5. Schematic layout of the CIRCE payloads within the lead and trail satellite buses. The UK's IRIS suite of payloads occupies the front 2 U of the leading satellite, and during night-time, the rear of the trailing satellite. The bus avionics are located in the centre $2 \mathrm{U}$ of each bus. The US Tri-TIP payloads occupy the rear $2 \mathrm{U}$ of the leading satellite, and during night-time, the front of the trailing satellite. This enables the Tri-TIP instruments to image the same volume of the ionosphere to support tomographic reconstruction. (Adapted from Nicholas et al., 2019, their Fig. 1).

direction. During transit of the day side, both IRIS instrument suites will operate in their optimal configurations. As both satellites transition back towards the night-side, the trail satellite will rotate $180^{\circ}$ once again. Both IRIS suites will continue to operate during transit of the night side. These regular manoeuvres mean that a few times a day, the TOPCAT II (GPS, see Sect. 4.2) and Tri-TIP (UV optical, see Sect. 2.2) instruments both have the same point of view, enabling direct data comparisons.

\section{IRIS payloads - detail}

\subsection{INMS}

The Ion and Neutral Mass Spectrometer (INMS, shown in Fig. 6) is a miniaturised cylindrical electrostatic particle analyser. INMS is designed for sampling low mass ionised and neutral particles in the spacecraft ram direction with the instrument resolutions optimised for resolving the major constituents in the lower thermosphere, i.e., $\mathrm{O}^{+}, \mathrm{O}_{2}, \mathrm{NO}$ and $\mathrm{N}_{2}$ ions and neutral particles.

The technology has previously been flown on TechDemoSat 2014 (see Fact Sheet at https://www.sa.catapult. org.uk), and QB50 missions (Thoemel et al., 2014). 11 INMS instruments were developed for the QB50 CubeSat constellation mission: 9 were launched on their respective educational CubeSats and to date, data has been returned over a six month period from the single working QB50 CubeSat. CIRCE will fly the two flight spare instruments from QB50, and is the only IRIS payload being flown as-is. Key components of the INMS flight model have a bespoke black Ebonol-C surface chemical conversion coating which is used to provide a better defined thermal performance and improve UV rejection (see Alsop et al., 1998).
The INMS has two electrostatically-selectable sensors with different instrument parameters. Each of the sensors consists of a collimator/ion filter, an ioniser and a charged particle analyser with a common charged particle detector at the exit of the analyser (see e.g. Fig. 5 in Agathanggelou et al., 2020). Charged particles entering the aperture can be rejected in the ion filter region by applying voltages to its electrodes. The ioniser consists of an electron source, an energy selector and a beam steerer and provides a beam of $50 \mathrm{eV}$ electrons that is steered into the charge exchange region. The current transmitted through is monitored by a Faraday cup at the exit of the region and can be actively controlled through a feedback loop.

The charged particle spectrometer consists of a cylindrical geometry analyser and a channel electron multiplier (CEM) detector. When voltages are applied to the ion filter and the ioniser is turned on, ions are rejected in the filter whereas the neutral particles are ionised and subsequently energy analysed in the analyser. On the other hand, when both are turned off, neutral particles pass through a gap in the analyser whereas the ions have their energy analysed in the analyser. The INMS can be operated alternatively in ion or neutral particle detection mode. With an energy resolution of $4 \%$, and $9 \%$ for the two sensing modes (ions and neutrals respectively), the analyser is designed to provide clean separation of the major constituents in both the upper and lower thermosphere. Table 2 details specific characteristics of INMS.

Figure 7 shows calibration data for the INMS flight model FM01 payload with the left panel showing the typical voltageelevation response for a $150 \mathrm{eV}$ beam and right panel showing the corresponding energy response extracted from the data.

It is anticipated that the neutral particle detection mode will be switched on at a later stage in the CIRCE mission. The initial operations will focus on sampling ionised particles. 

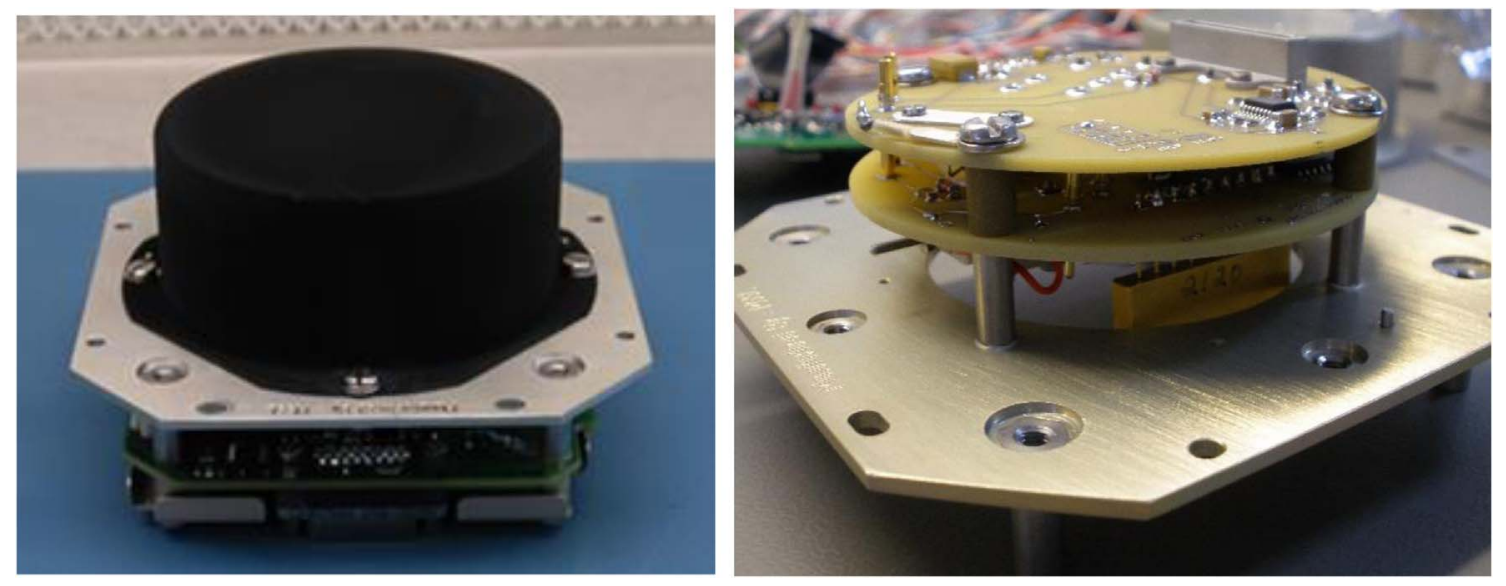

Fig. 6. INMS flight model: with the black Ebonol-C coated cylindrical top hat (left), and without (right), showing the flight digital boards (courtesy UCL/MSSL).

Table 2. Characteristics of INMS.

\begin{tabular}{lc}
\hline Particles detected & Ions/neutrals \\
\hline$K$ factor & 2.52 \\
Geometric factor & $6 \times 10^{-4} \mathrm{~cm}^{2} \mathrm{sr}(\mathrm{eV} / \mathrm{eV})$ \\
Energy resolution & $0.04 / 0.09$ \\
Energy acceptance & $0.28-70 \mathrm{eV}$ \\
Angular resolution & $1.3^{\circ} \times 2.0^{\circ}$ \\
Field of view steering & Electrostatic \\
Angular acceptance & $1.3^{\circ} \times 7.0^{\circ}$ \\
\hline
\end{tabular}

\subsection{TOPCAT II}

Two new tri-band GPS receiver payloads, TOPside ionosphere and plasmasphere Computer Assisted Tomography, (TOPCAT II), will allow remote measurement of electron densities between LEO satellites and GPS satellites to improve the vertical resolution of current ionospheric imaging. TOPCAT II will operate at the GPS L1 band (centre frequency 1575.42 MHz), L2 band (centre frequency $1227.60 \mathrm{MHz}$ ), and L5 band (centre frequency $1176.45 \mathrm{MHz}$ ), at $1 \mathrm{~Hz}$ standard, with a high data rate of $10 \mathrm{~Hz}$. Figure 8 shows a photograph of a TOPCAT II flight model.

Ionospheric total electron content (TEC) tomography utilises the integral of the number of free electrons between satellite and receiver, measured by radio signals propagating through the medium. For GPS-based tomography, TEC measurements are derived from the differential phase of dualfrequency transmissions of GPS satellites. The final TEC allows four-dimensional (4-D - i.e. 3-D spatial and time evolving) mapping of the ionosphere (Bust \& Mitchell, 2008).

The TOPCAT II payload consists of a GPS receiver, a payload controller board, and a triple frequency stacked patch antenna. The TOPCAT II patch antenna is connected to the GPS receiver via a mini-bend radio frequency cable. The TOPCAT II payloads were successfully qualified for operating in a LEO environment through a rapid development and series of ground testing from February to July 2019. Phase data collected by the receiver will be used to image the electron density of the ionosphere and plasmasphere using tomography and data assimilation from the University of Bath MultiInstrument Data Assimilation System (MIDAS) algorithms (Mitchell \& Spencer, 2003).

TOPCAT I was installed on-board the UK Space Agency's UKube-1, launched 2014 (Pinto-Jayawardena, 2015). Although TOPCAT II draws on this heritage, the TOPCAT II payload required an entirely new electronic design due to a new GPS receiver (the previous version was no longer in production). The ability to receive L5 GPS frequencies is a novel aspect for the CIRCE mission.

The TOPCAT II antenna and low-noise amplifier (LNA) module is powered via a DC bias over the coaxial cable from the TOPCAT II receiver module. The stacked patch antenna has an approximately hemispherical radiation pattern with the peak antenna gain in the direction normal to the ground plane. The antenna is closely coupled into a low-noise amplifier and associated bandpass filters before being combined into a single SMA (sub-miniature type-A) connection. The internal antenna elements and bandpass filters provide selection of signals at L1 (centre frequency $1575.42 \mathrm{MHz}$ ) and L2/L5 (centre frequencies of $1227.60 \mathrm{MHz}$ and $1176.45 \mathrm{MHz}$, respectively).

Figure 9 shows the measurement of the antenna and LNA gain and the output return loss of the antenna. Measurement was made using a vector network analyser using a test antenna.

With reference to the gain trace (red), it can be seen that there is good selection of the L1/L2/L5 bands and strong rejection of signals outside of these frequency bands. The blue trace shows the return loss at the output. This is below $-15 \mathrm{~dB}$ across the whole band of interest which indicates that the antenna is well matched to the TOPCAT II antenna input.

\subsection{RadMon}

The CIRCE/IRIS radiation monitor (RadMon) comprises: a particle detector, dose rate monitor and total dose monitor. It measures high energy proton fluxes, heavy ion linear energy transfer spectra, ionisation photocurrent, and total dose (internally and in up to 3 external locations). The particle energy bands are linearly spaced at approximately $0.05 \mathrm{MeV} \mathrm{cm} \mathrm{mg}^{-1}$ per band. As well as its on-board RadFET dosimeter, it can connect to up to three remote RadFETs, increasing redundancy and 

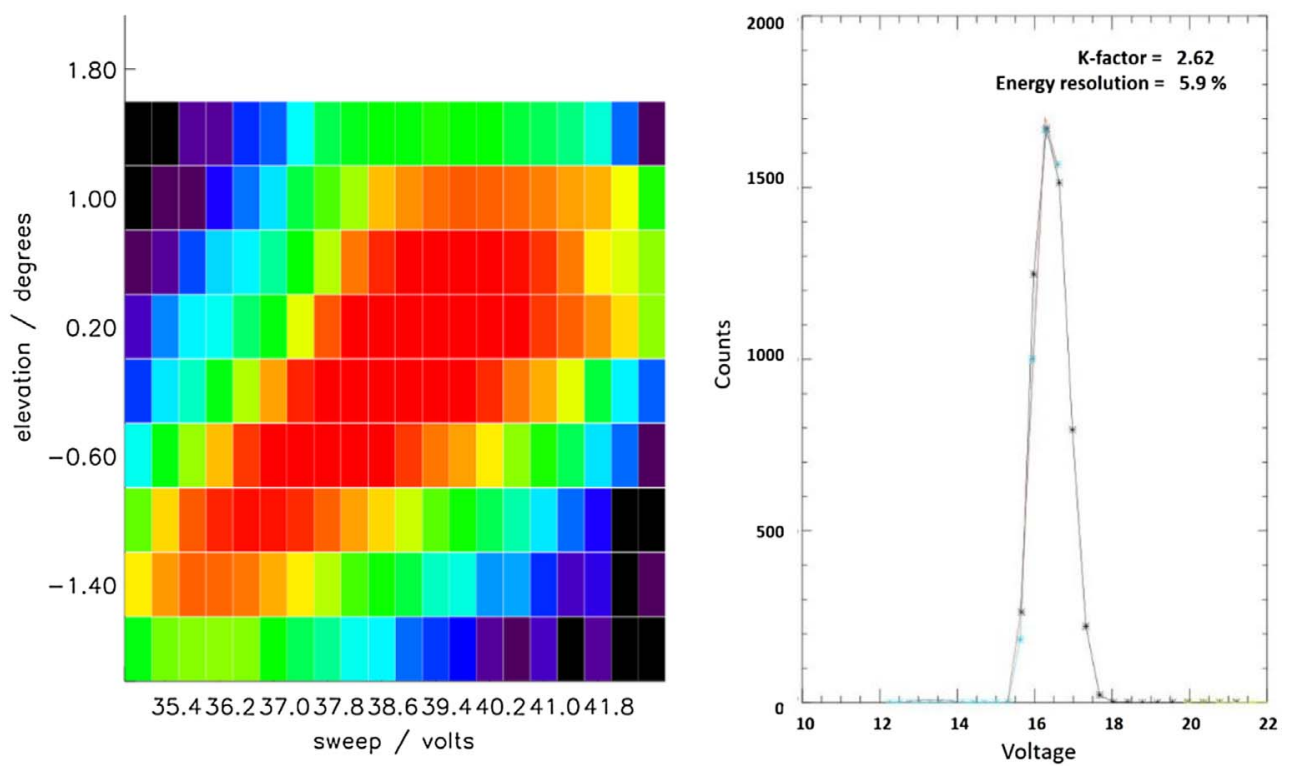

Fig. 7. Calibration data for the INMS flight model FM01 payload. Left panel shows the typical voltage-elevation response and right panel shows the corresponding energy response.

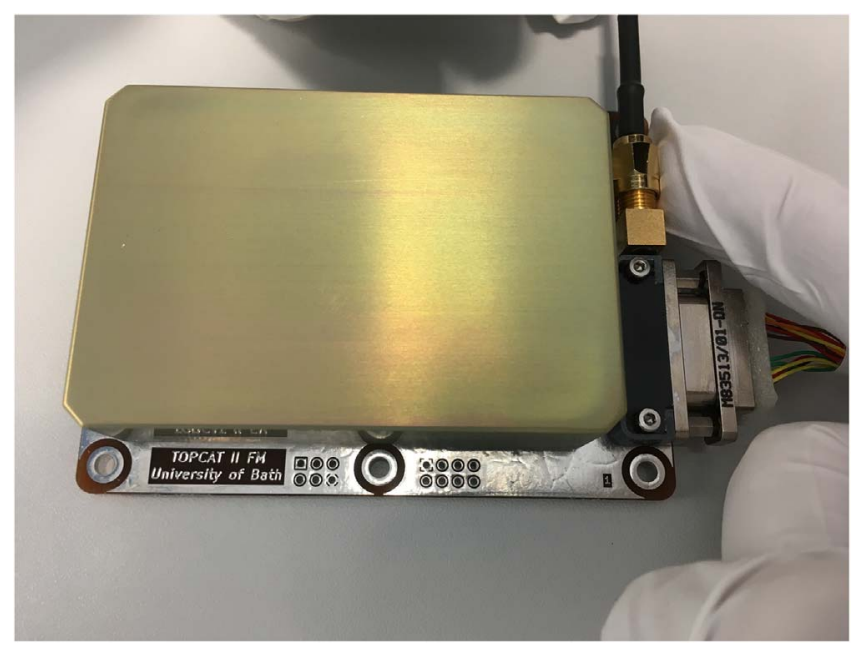

Fig. 8. TOPCAT II flight model. The shield protects the GPS receiver and electronic printed circuit board (courtesy University of Bath).

allowing the operator to build up a picture of radiation throughout the spacecraft. The external RadFETs have been utilised within each IRIS suite, with two sets mounted on a single board, and the third set located on the opposite side of the IRIS clamshell.

If a spacecraft is not sufficiently shielded from the harsh radiation environment, delicate electronics on board can be degraded over time, leading to reduced mission lifetimes and premature critical failures. One approach to avoiding these issues is to build up maps of the various radiation environments to enable improved prediction of how any given spacecraft or system will be affected. To do this, measurements can be taken in situ by spacecraft already in orbit, generating the need for payloads designed specifically for this task.

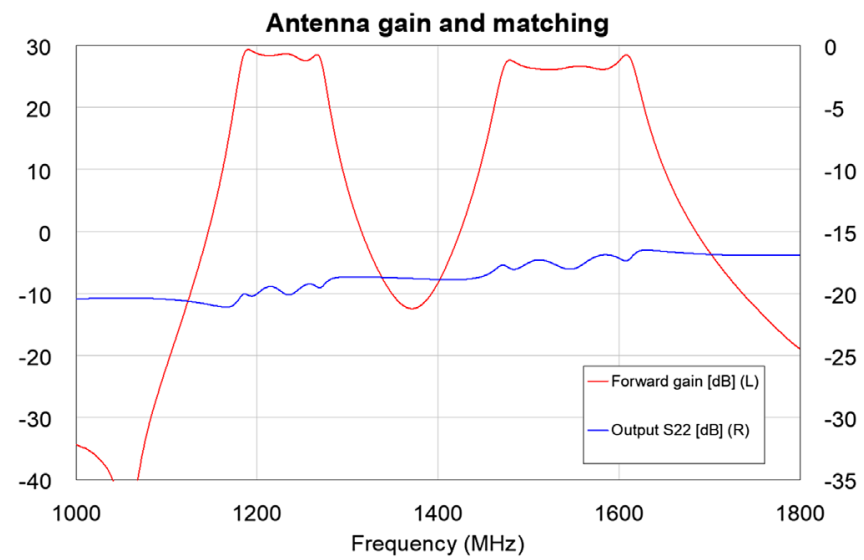

Fig. 9. Showing the performance of the bandpass filter and amplifiers within the TOPCAT II stacked patch antenna. The gain trace (red) shows a good selection of the L1/L2/L5 bands, and strong rejection of signals outside of these frequency bands. The blue trace shows the return loss at the output.

For LEO satellites, a significant space weather event will cause expansion and contraction (due to heating and cooling, respectively) of the Earth's atmosphere causing variable drag conditions (e.g. for heating, the denser atmosphere is expanded to higher altitudes). The result is that orbits will be disturbed and predictions of satellite positions will be degraded. The March 1989 space weather event drove the Solar Maximum Mission satellite (in LEO) to drop $500 \mathrm{~m}$ at the start of the storm, and over $5 \mathrm{~km}$ drop overall (e.g. Kennewell, 1999). This has implications for satellite tracking, re-entry epochs and satellite conjunctions - all of which become harder to manage for constellations. After the initial disruption, satellite orbit data then needs to be re-acquired which may take some days to complete. In extreme cases, low altitude satellites may experience 
significant aerodynamic torques which overcome the vehicle's attitude control system capability leading to termination of the mission (Cannon et al., 2013).

In addition to dynamic space weather impacts, another wellknown source of radiation impacts to satellites includes the significant elevations of radiation dose rate experienced by a satellite operating in LEO transiting the South Atlantic Anomaly (SAA). The SAA is an area of low surface geomagnetic field intensity that spans the southern Atlantic Ocean, Africa, and South America. It is so pronounced that it allows the close approach of Earth's inner radiation belt, creating an ionospheric feature known as the SAA (Tarduno et al., 2015). For LEO, the spacecraft typically passes through the SAA in approximately $20 \mathrm{~min}$.

There are six SSTL-developed radiation monitors in orbit, providing a near continuous data set from 1992. The latest iteration for CIRCE (RadMon 3.0), now features a new more powerful microcontroller with the detector control circuitry further optimised to ensure the RadFETs are always operated in their optimum configuration even with no power applied to the module. The more powerful microcontroller captures higher resolution detail for radiation particle events and records data over many hours, minimising the number of data collection visits required by the spacecraft on board computer. RadMon maintains over 80 telemetry points for monitoring health and power demand of the unit plus background radiation dose rate, particle hit rate, total dose plus flare event statistics. RadMon also supports a streaming communications interface for transfer of particle hit counts recorded and saved in ferroelectric random access memory.

Responding to the dynamic space environment theme of CIRCE, an on-orbit configurable radiation flare event detection warning is also incorporated in the latest design. Flare event detection is required during the first few minutes of rising dose rate. Both dose rate diode-voltage (DRD-V) and particle hit detection methods are employed for RadMon 3.0. These may be enabled by telecommand to operate either singularly or together. The detection works by setting thresholds for the requisite level above the background average, plus gradient. Telemetries are maintained for the background average, and the current and maximum values detected for each sensor.

RadMon 1.0 was already a compact, lightweight unit, with a mass of $700 \mathrm{~g}$ and dimensions of $100 \mathrm{~mm} \times 70 \mathrm{~mm} \times 65 \mathrm{~mm}$ including its own shielding metalwork. However by utilizing a new, more powerful microcontroller and partitioning the circuitry into "noisy" and "sensitive" parts, the number of printed circuit assemblies has been halved from four to two, and the overall size of the unit has been reduced by $40 \%$ (see Figs. 10 and 11). The new layout required alterations to the existing circuit board design (though wherever possible the alterations draw heavily on existing heritage). Two flight models of the latest version, RadMon 3.0, have been built for CIRCE/IRIS, and have successfully completed full environmental and functional test campaigns.

The RadFETs used are all from the same batch. Each IRIS module contains four separate RadFETs, and as each RadFET device contains two detectors, four co-sited pairs. This allows cross-calibration between the dose rate information from the individual detectors on the same spacecraft and also between the two spacecraft. All the devices have the same characteristic $\Delta V$ vs. total dose curve but each device has a different initial

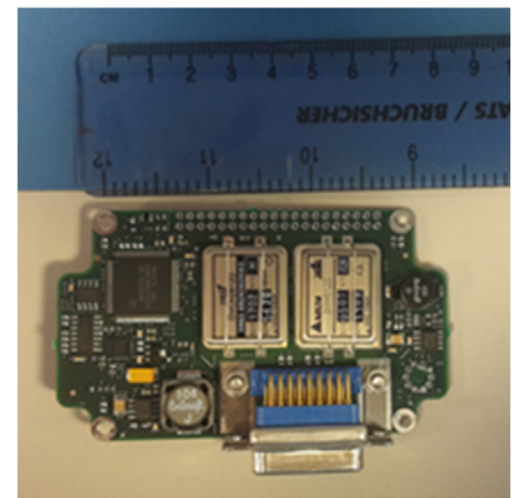

Fig. 10. Showing a RadMon 3.0 engineering model, for CIRCE (courtesy SSTL).

offset. The differing initial offsets are due to slight variations in the threshold voltage of each individual FET that make up the eight independent total ionising dose detectors, caused by normal manufacturing variations. The characteristics for the devices fitted to RadMon flight model FM1 are plotted in the left hand panel of Figure 12 and for flight model FM2 in the right hand panel of Figure 12.

\subsection{IRIS mechanical design}

The IRIS chassis is a three-part clamshell, custom designed to accommodate all three IRIS payloads (including the TOPCAT II antenna), whilst still fitting within the designated envelope of the spacecraft (see Fig. 13). It is made from aluminium alloy $2 \mathrm{~mm}$ thick to provide suitable radiation shielding for the mission orbit and duration, and it is treated with an Alocrom 1200 coating to protect it prior to launch. Surfaces that will be exposed once the chassis is fitted within the spacecraft are covered with secondary surface mirrors to aid the thermal case.

The clamshell is three parts rather than two to aid the assembly process by eliminating any deep pockets that would hinder the securing of modules and harness. At the rear of the unit is an aperture for harness that matches that of the spacecraft. The TOPCAT II antenna is mounted on the opposing face, which also features an aperture to fit INMS. The height of the chassis is dictated by the height of these units, so they are kept close to the envelope of the spacecraft to maximise their performance by providing adequate exposure to the environment. The chassis is secured by six titanium bolts, which allow a preload necessary to keep IRIS secure throughout the vibration and shock environments endured during launch and deployment. Figure 14 shows a photograph of one of the IRIS flight units, complete with the IRIS payloads.

\section{IRIS data exploitation}

The overarching goal is to exploit the data returned from CIRCE to enhance ionospheric nowcast and forecasting capabilities. We are aiming to demonstrate the specific benefit of data-driven local/regional ionospheric predictions, and their utility in producing accurate ray-tracing, data products, and decision aids. 


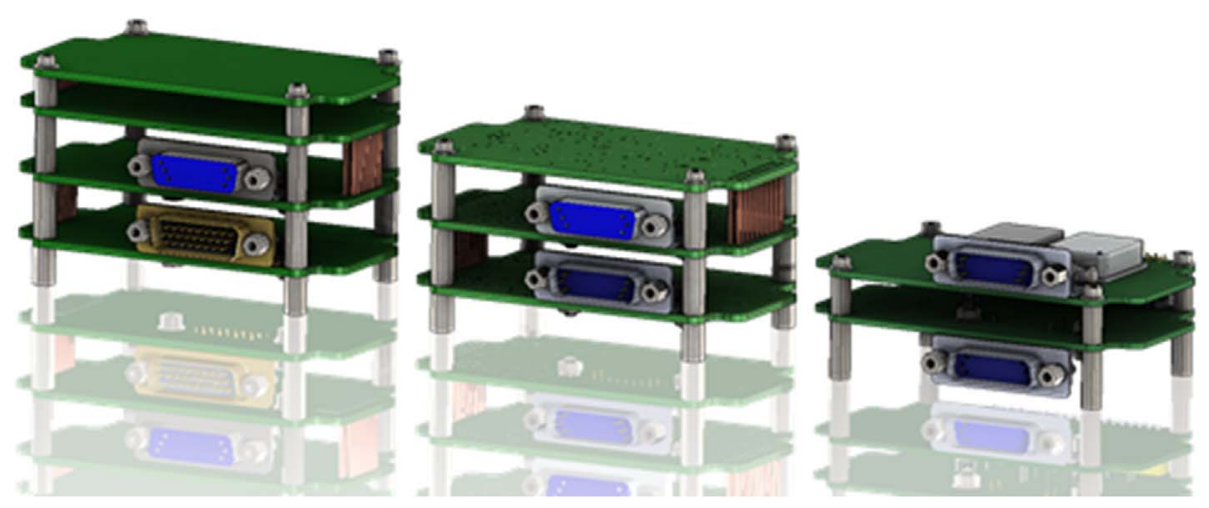

Fig. 11. Illustrating the relative reductions in size for RadMons 1.0, 2.0, and 3.0 (for CIRCE). Figure courtesy SSTL.
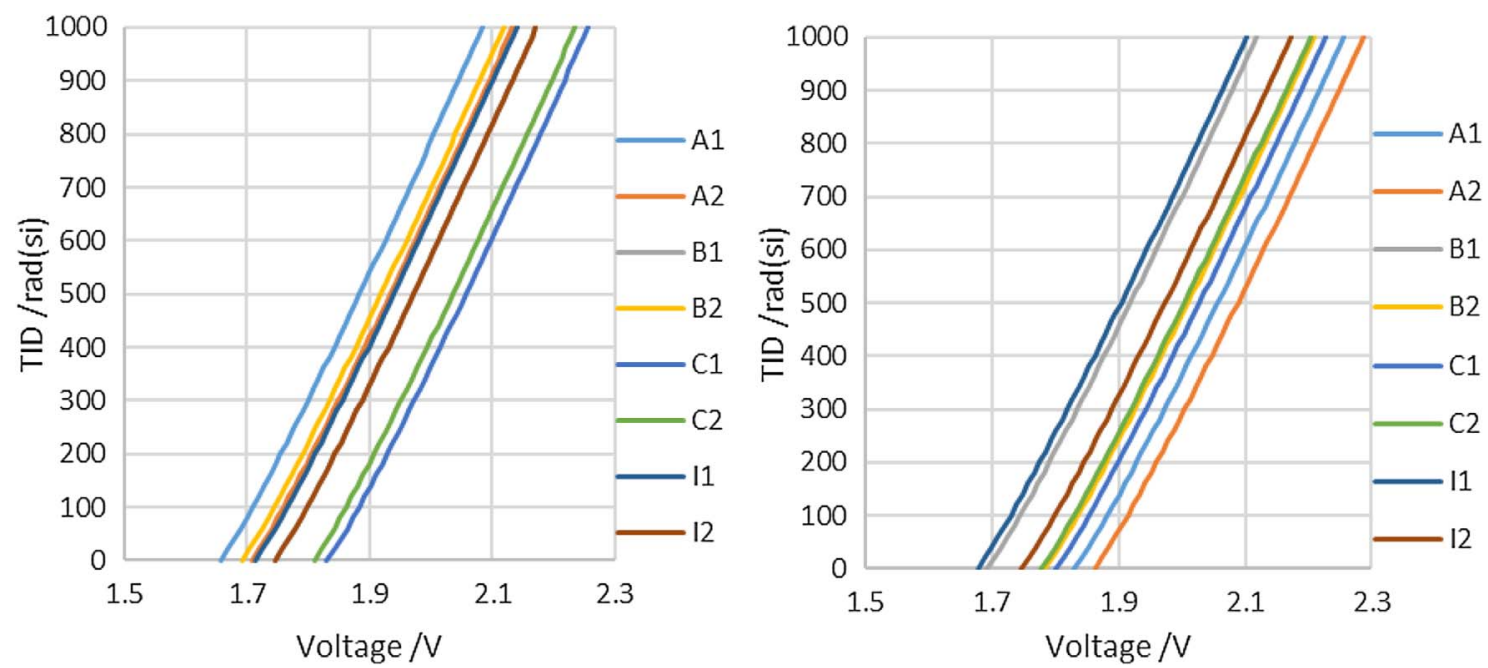

Fig. 12. RadFET voltage vs. total received dose for RadMon flight models FM1 and FM2.

It is anticipated that data from IRIS, specifically INMS, will improve our understanding of the variability of atmospheric drag, the chemistry of the thermosphere and the impact of space weather on the upper-atmosphere. Key information regarding the particle species at the thermosphere-ionosphere lower boundary is critical for the refinement of data assimilation models, e.g. the physics-based Advanced Ensemble electron density Assimilative System (AENeAS; Elvidge \& Angling, 2019). AENeAS has been designed with a modular architecture, specifically with a view to incorporating data from new sources.

Data returned from TOPCAT II will be used to validate the Multi-Instrument Data Analysis System (MIDAS; Mitchell \& Spencer, 2003) tomography algorithm for the topside ionosphere and plasmasphere, by using total electron content (TEC) measurements from the differential phase of GPS signals, and inverting them to derive the electron density of the region.

The data returned from the RadMon instruments will identify areas of increased radiation for satellites to avoid, and help identify suitable orbits/shielding requirements for future satellites. Data associated with the flare event detection trigger, developed for CIRCE, will be used to evaluate performance impacts on other instruments as a result of charged particle events.
In addition to the results anticipated from the individual CIRCE payloads, there is a wealth of synergistic science that can be derived from the complement of ten instruments onboard the two CIRCE satellites. Examples include opportunities for cross-calibration of the sensors between the buses at an early stage in the mission (when located closest together). Further potential scientific studies include utilising INMS (relative, or absolute with good calibration) in situ ion density measurements in support of tomography; comparisons of TOPCAT II data detecting scintillation with Tri-TIP UV image reconstruction of bubbles; and using RadMon data to characterize noise on the INMS micro-channel plates.

Although the primary function of CIRCE data will be exploited for scientific benefit, it can also be utilised for engineering purposes (see Honig et al., 2019, for other examples of this type of dual functionality). For example, the RadMon capability will monitor, in near real-time, the rates of highly energetic particles that can cause damage to spacecraft components. This information can inform operational decisions for both the payloads and the satellite buses.

The data returned from CIRCE will initially be restricted to the CIRCE science team lead by Dstl and NRL. The payload owners will have first access to the data during the 


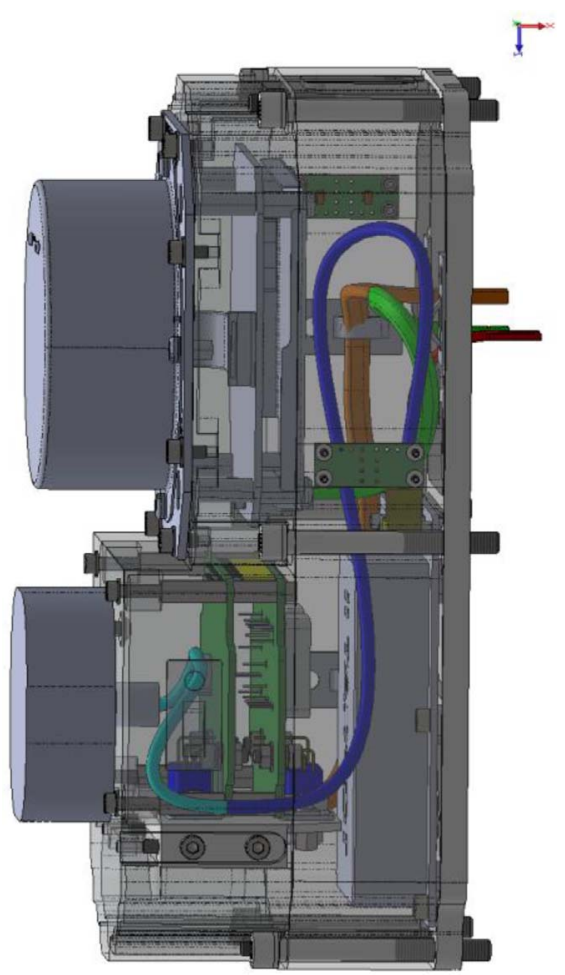

Fig. 13. Computer aided design (CAD) model showing how the IRIS payloads are secured within the three-part clam-shell structure.

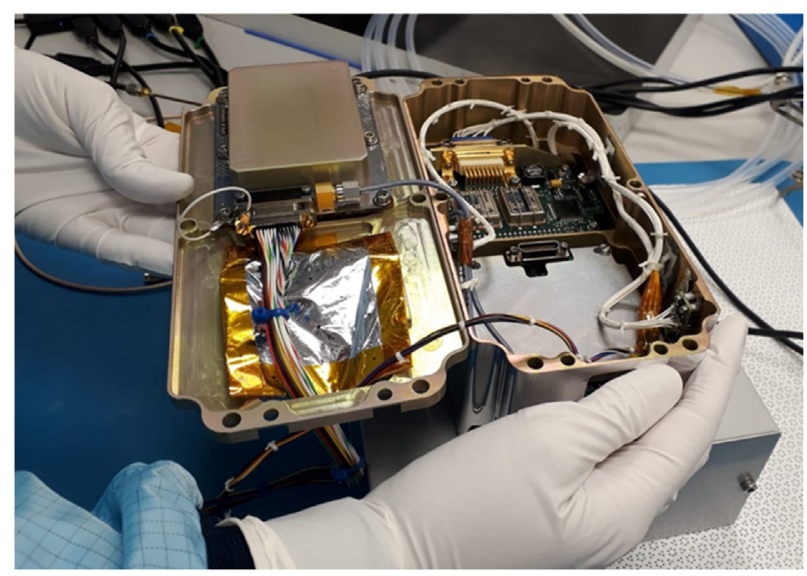

Fig. 14. Photograph showing one of the IRIS flight units, complete with integrated INMS, TOPCAT II, and RadMon, whilst at SSTL. The pass-thru to the bus avionics section is temporarily covered by multi-layer insulation (MLI-Mylar).

commissioning phase. This will be then be extended to the full CIRCE science team during nominal operations, which is due to last for a period of one year. The CIRCE data will be archived, and later be made available to external third parties.

\section{Summary}

CIRCE/IRIS comprises the following payload compliment:
- Two (1 per Cubesat) Ion and Neutral Mass Spectrometers (INMS).

- Two (1 per Cubesat) Topside ionosphere computer assisted tomography (TOPCAT II) triple-frequency GPS receivers.

- Two (1 per Cubesat) Radiation Monitors (RadMon) for particle detection, dose rate, and total dose.

In addition:

- Four (2 per Cubesat) Triple Tiny Ionospheric Photometers (Tri-TIP) UV $135.6 \mathrm{~nm}$ Oxygen emission, are provided by NRL.

The combination of these instruments means that considerable scientific capability ( 5 payloads per satellite) is packed into each 6U CubeSat for the collaborative CIRCE mission. All three IRIS payloads occupy a small volume no more than $2 \mathrm{U}$ $(10 \mathrm{~cm} \times 10 \mathrm{~cm} \times 20 \mathrm{~cm})$. This type of instrument miniaturisation could encourage the deployment of many more such space environment sensors as secondary payloads, becoming a routine inclusion on other satellites due to their small size, weight and power. The result would expand data collection for the near-Earth space environment, and significantly enhance space environment modelling capabilities.

Acknowledgements. The authors sincerely thank the referees for their thoughtful comments and helpful suggestions. The CIRCE mission is a jointly-funded programme between the UK Defence Science and Technology Laboratory and the US Naval Research Laboratory. The UK's IRIS payloads acknowledge funding from Dstl's space programme. Work at NRL for all authors is supported by the Chief of Naval Research. We acknowledge with thanks the work of the team at Blue Canyon Technologies. Content includes material subject to ${ }^{\circ}$ Crown copyright (2020), Dstl. This material is licensed under the terms of the Open Government Licence except where otherwise stated. To view this licence, visit http:// www.nationalarchives.gov.uk/doc/open-government-licence/ version/3 or write to the Information Policy Team, The National Archives, Kew, London TW9 4DU, or email: psi@ nationalarchives.gov.uk. The editor thanks two anonymous reviewers for their assistance in evaluating this paper.

\section{References}

Agathanggelou AP, Attrill GDR, Nicholas AC, Routledge GJ, Miah JA, et al. 2020. CIRCE: Coordinated Ionospheric Reconstruction Cubesat Experiment. In: Proceedings of the Small Satellite Conference, Science/Mission Payloads, Utah State University. SSC20-III-02. https://digitalcommons.usu.edu/smallsat/2020/all2020/117/.

Alsop C, Scott S, Free L. 1998. UV rejection design and performance of the PEACE electrostatic analyzers. In: Measurement techniques in space plasmas: Particles, 102, AGU monograph series. https://doi.org/10.1029/GM102p0269.

Altadill D, Segarra A, Estefania B, Juan Miguel, Paznukhov V, et al. 2020. A method for real-time identification and tracking of traveling ionospheric disturbances using ionosonde data: First results. J Space Weather Space Clim 10: 2. https://doi.org/ $10.1051 /$ swsc/2019042. 
Basu S, Groves KM. 2001. Specification and forecasting of outages on satellite communication and navigation systems. Space Weather Geophys Monogr 125: 423. https://doi.org/10.1029/GM125p0423.

Basu S, MacKenzie E, Basu Su. 1988. Ionospheric constraints on VHF/UHF communication links during solar maximum and minimum periods. Radio Sci 23: 363. https://doi.org/10.1029/ RS023i003p00363.

Beggan CD, Beamish D, Richards A, Kelly GS, Thomson AWP. 2013. Prediction of extreme geomagnetically induced currents in the UK high-voltage network. Space Weather 11: 407-419. https://doi.org/10.1002/swe.20065.

Bust GS, Mitchell CN. 2008. History, current state, and future directions of ionospheric imaging. Rev Geophys 46(1). https://doi. org/10.1029/2006RG000212. https://agupubs.onlinelibrary.wiley.com/doi/full/10.1029/2006RG000212.

Cannon P, Angling M, Barclay L, Curry C, Dyer C, et al. 2013. Extreme space weather: Impacts on engineered systems and infrastructure. Royal Academy of Engineering, London, ISBN 1-903496-95-0.

Dellinger JH. 1937. Sudden disturbances of the ionosphere. J Appl Phys 8(11): 732. https://doi.org/10.1063/1.1710251.

Dungey JW. 1961. Interplanetary magnetic field and the auroral zones. Phys Rev Lett 6: 47-48. https://doi.org/10.1103/physrevlett.6.47.

Elvidge S, Angling MJ. 2019. Using the local ensemble transform Kalman filter for upper atmospheric modelling. J Space Weather Space Clim 9: A30. https://doi.org/10.1051/swsc/2019018.

Hapgood M, Gopalswamy N, Leka KD, Barnes G, Yermolaev Yu, et al. 2018. Extreme events in geospace: Origins, predictability, and consequences. Elsevier. ISBN 978-0-12-812700-1. https://doi. org/10.1016/C2016-0-03769-5.

Hargreaves JK. 1992. The solar-terrestrial environment: an introduction to geospace - the science of the terrestrial upper atmosphere, ionosphere and magnetosphere. Cambridge University Press, Cambridge, UK.

Honig T, Witasse OG, Evans H, Nieminen P, Kuulkers E, et al. 2019. Multi-point galactic cosmic ray measurements between 1 and 4.5 AU over a full solar cycle. Ann Geophys 37: 903-918. https://doi. org/10.5194/angeo-37-903-2019.

Kennewell J. 1999. Satellite orbital decay calculations. IPS Radio and Space Services, The Australian Space Weather Agency, Australian Government Bureau of Meterorology, Sydney, Australia.
Lockwood M. 2016. Jim Dungey, The open magnetosphere, and space weather. Space Weather 14: 380-383. https://doi.org/ 10.1002/2016SW001438.

Mitchell CN, Spencer PSJ. 2003. A three-dimensional timedependent algorithm for ionospheric imaging using GPS. Ann Geophys 46(4): 687-696. https://doi.org/10.4401/ag-4373.

Nicholas AC, Attrill GDR, Dymond KF, Budzien SA, Stephan AW, et al. 2019. Coordinated ionospheric reconstruction CubeSat experiment (CIRCE) mission overview. In: Proc. SPIE 11131, CubeSats and SmallSats for Remote Sensing III, 111310E p. https://doi.org/10.1117/12.2528767.

Nita GM, Gary DE, Lanzerotti LJ, Thomson DJ. 2002. The peak flux distribution of solar radio bursts. ApJ 570(1): 423. https://doi.org/ $10.1086 / 339577$.

Phillips KJH. 1992. Guide to the sun. Cambridge University Press, Cambridge, MA.

Pick M. 2006. Radio emissions from the sun and the interplanetary medium. In: Solar and heliospheric origins of space weather phenomena, Rozelot J-P (Ed.), Vol. 699 of Lecture Notes in Physics,Springer Verlag, Berlin, 119 p. https://doi.org/10.1007/3540-33759-8_6.

Pinto-Jayawardena T. 2015. Topside ionosphere/plasmasphere tomography using space-borne dual frequency GPS receivers. Ph.D., University of Bath, Bath, UK. https://researchportal.bath. ac.uk/en/studentTheses/topside-ionosphereplasmasphere-tomography-using-space-borne-dual-.

Schunk RW, Nagy A. 2009. Ionospheres: Physics, plasma physics, and chemistry, Cambridge atmospheric and space science series. Cambridge University Press, Cambridge, UK. https://doi.org/ 10.1017/CBO9780511635342.

Solomon SC, Roble RG. 2015. Thermosphere. In: Encyclopedia of atmospheric sciences (2nd ed., North GR, Pyle J, Zhang F (Eds.), Academic Press, pp. 402-408. ISBN 9780123822253. https://doi. org/10.1016/B978-0-12-382225-3.00408-4.

Tarduno JA, Watkeys MK, Huffman TN, Cottrell RD, Blackman EG, et al. 2015. Antiquity of the South Atlantic Anomaly and evidence for top-down control on the geodynamo. Nat Commun 6: 7865. https://doi.org/10.1038/ncomms8865.

Thoemel J, Singarayar F, Scholz T, Masutti D, Testani P, et al. 2014. Status of the QB50 CubeSat constellation mission. In: 65th International Astronautical Congress., Toronto, Canada, IAC-14-B4.2.11.

Cite this article as: Attrill GDR, Nicholas AC, Routledge G, Miah JA, Kataria DO, et al. 2021. Coordinated Ionospheric Reconstruction CubeSat Experiment (CIRCE), In situ and Remote Ionospheric Sensing (IRIS) suite. J. Space Weather Space Clim. 11, 16. https://doi.org/ $10.1051 / \mathrm{swsc} / 2020066$. 